\title{
Sign-balances of Tableaux with at Most Three Rows
}

\author{
Tung-Shan Fu and Chien-Tai Ting*
}

\begin{abstract}
Let the sign of a standard Young tableau (SYT) be the sign of the permutation obtained by reading the entries row by row from left to right, starting from the top row. By a sign-reversing involution on the tableaux in terms of lattice paths, we obtain the sign-balance of $n$-cell SYTs of all shapes with at most three rows. For skew shapes, we obtain partial results on the sign-balance enumeration of $2 n$-cell skew SYTs of all shapes with at most three rows, under a parity condition of the skew part.
\end{abstract}

\section{Introduction}

\subsection{The sign of a tableau}

The shape of an integer partition $\lambda=\left(\lambda_{1}, \lambda_{2}, \ldots\right)$ of $n$ is a left-justified array of cells with $\lambda_{i}$ cells in the $i$ th row. For a partition $\mu=\left(\mu_{1}, \mu_{2}, \ldots\right)$ with $\lambda_{i} \geq \mu_{i}$ for all $i$, the skew shape of $\lambda / \mu$ is the array obtained by removing the cells of $\mu$ from $\lambda$. An $n$-cell skew standard Young tableau $T$ is a filling of a shape $\lambda / \mu$ with entries $\{1,2, \ldots, n\}$, increasing along rows and columns. If the skew part $\mu$ is empty then $T$ is called a standard Young tableau (SYT). The sign of a tableau $T$, denoted by $\operatorname{sign}(T)$, is defined to be $(-1)^{\operatorname{inv}(\sigma(T))}$, where $\sigma(T)$ is the permutation obtained from $T$ by reading the entries row by row from left to right, starting from the top row. For example, the permutation obtained from the tableau $T$ shown below is $\sigma(T)=13625748$, which has 6 inversions, and hence $\operatorname{sign}(T)=+1$.

$$
T=\begin{array}{|l|l|l|}
\hline 1 & 3 & 6 \\
\hline 2 & 5 & 7 \\
\hline 4 & 8 &
\end{array} \quad \sigma(T)=13625748 .
$$

Stanley conjectured that the sign-balance of all $n$-cell SYTs is $2^{\lfloor n / 2\rfloor}$. This conjecture was proved independently by Lam [3], Reifegerste [5] and Sjöstrand [7]. For skew shapes, Sjöstrand [8] obtained analogous sign-balance identities by the skew Robinson-Schensted correspondence invented by Sagan and Stanley [6]. Lam [4] gave an alternative proof of Sjöstrand's skew sign-balance results. In this paper, we study the sign-balance of $n$-cell (skew) SYTs of all shapes with at most three rows.

Received February 5, 2021; Accepted March 14, 2021.

Communicated by Sen-Peng Eu.

2020 Mathematics Subject Classification. Primary: 05A15; Secondary: 05A19.

Key words and phrases. sign-balance, standard Young tableaux, standard domino tableaux, Motzkin paths.

*Corresponding author. 


\subsection{Main results}

Let $\mathcal{T}_{n}$ denote the set of $n$-cell SYTs of all shapes with at most three rows. It is well known that the cardinality of $\mathcal{T}_{n}$ is the $n$th Motzkin number $m_{n}$. The Motzkin numbers $\left\{m_{n}\right\}_{n \geq 0}=\{1,1,2,4,9,21,51, \ldots\}$ can be defined by $m_{0}=1$ and $m_{n}=m_{n-1}+$ $\sum_{k=2}^{n} m_{k-2} m_{n-k}$ for $n \geq 1$ [9, A001006]. One of our main results is that the sign-balance of the set $\mathcal{T}_{2 n}\left(\mathcal{T}_{2 n+1}\right.$, respectively) of SYTs can be expressed as a partial sum of the sequence of Motzkin numbers.

Theorem 1.1. For $n \geq 0$, the following results hold.

(i) $\sum_{T \in \mathcal{T}_{2 n}} \operatorname{sign}(T)=1+m_{0}+m_{1}+\cdots+m_{n-1}$.

(ii) $\sum_{T \in \mathcal{T}_{2 n+1}} \operatorname{sign}(T)=1+m_{0}+m_{1}+\cdots+m_{n-1}$.

The proof of Theorem 1.1 is accomplished by a sign-reversing involution on the tableaux, on the basis of a previously established bijection between standard domino tableaux and partial Motzkin paths by Cheng et al. in [2].

The generating polynomial for Motzkin numbers $\left\{m_{n}\right\}_{n \geq 0}$ is

$$
M=M(x)=\sum_{n \geq 0} m_{n} x^{n}=\frac{1-x-\sqrt{1-2 x-3 x^{2}}}{2 x^{2}} .
$$

Notice that the sign-balance results in Theorem 1.1 can be expressed in the form of the generating polynomial as

$$
\sum_{n \geq 0}\left(\sum_{T \in \mathcal{T}_{2 n}} \operatorname{sign}(T)\right) x^{n}=\sum_{n \geq 0}\left(\sum_{T \in \mathcal{T}_{2 n+1}} \operatorname{sign}(T)\right) x^{n}=\frac{1+x M}{1-x}
$$

For skew shapes, let $\mathcal{T}_{n}^{\left(\mu_{1}, \mu_{2}\right)}$ denote the set of $n$-cell skew SYTs of all shapes with at most three rows and the skew part $\mu=\left(\mu_{1}, \mu_{2}\right)$. We consider the $2 n$-cell skew SYTs. The enumerations of sign-balance of the initial sets $\mathcal{T}_{2 n}^{\left(\mu_{1}, \mu_{2}\right)}$ are listed below for several skew shapes $\mu=\left(\mu_{1}, \mu_{2}\right)$.

We obtain partial results on the enumeration of sign-balance of $\mathcal{T}_{n}^{\left(\mu_{1}, \mu_{2}\right)}$, under a parity condition of $\mu_{1}$ and $\mu_{2}$. We make use of the $\chi$-notation that maps each statement $P$ onto $\{0,1\}$, defined as $\chi(P)=1$ if $P$ is true, and 0 otherwise. 


\begin{tabular}{|c|c|c|c|c|c|c|c|c|c|c|}
\hline$\mu$ & $n:$ & 1 & 2 & 3 & 4 & 5 & 6 & 7 & 8 & 9 \\
\hline$(0,0)$ & & 2 & 3 & 5 & 9 & 18 & 39 & 90 & 217 & 540 \\
\hline$(1,1)$ & & 2 & 3 & 5 & 13 & 28 & 67 & 160 & 397 & 1002 \\
\hline$(2,0)$ & & 3 & 6 & 12 & 25 & 55 & 127 & 305 & 755 & 1913 \\
\hline$(3,1)$ & & 2 & 5 & 11 & 25 & 58 & 149 & 370 & 945 & 2472 \\
\hline$(3,2)$ & & 2 & 5 & 11 & 25 & 58 & 139 & 342 & 861 & 2208 \\
\hline$(3,3)$ & & 3 & 6 & 12 & 25 & 65 & 155 & 389 & 991 & 2555 \\
\hline$(4,1)$ & & 2 & 4 & 9 & 21 & 51 & 127 & 323 & 835 & 2188 \\
\hline$(4,2)$ & & 3 & 9 & 22 & 53 & 128 & 315 & 790 & 2017 & 5229 \\
\hline
\end{tabular}

Table 1.1: The sign-balances of $\mathcal{T}_{2 n}^{\left(\mu_{1}, \mu_{2}\right)}$.

Theorem 1.2. For $e_{1} \geq e_{2} \geq 0$, the following results hold.

(i) We have

$$
\begin{aligned}
\sum_{n \geq 0}\left(\sum_{T \in \mathcal{T}_{2 n}^{\left(2 e_{1}, 2 e_{2}\right)}} \operatorname{sign}(T)\right) x^{n}= & \sum_{a=1}^{e_{2}} \sum_{b=1}^{e_{1}-e_{2}} x^{e_{1}-a-b} M^{e_{1}-a-b+1} \\
& +\sum_{a=0}^{e_{2}} \sum_{b=0}^{e_{1}-e_{2}} \chi(a b=0) x^{e_{1}-a-b} M^{e_{1}-a-b} \frac{(1+x M)}{1-x}
\end{aligned}
$$

(ii) We have

$$
\begin{aligned}
& \sum_{n \geq 0}\left(\sum_{T \in \mathcal{T}_{2 n}^{\left(2 e_{1}+1,2 e_{2}\right)}} \operatorname{sign}(T)\right) x^{n} \\
= & \sum_{a=1}^{e_{2}} \sum_{b=0}^{e_{1}-e_{2}} x^{e_{1}-a-b} M^{e_{1}-a-b+1}+\sum_{b=0}^{e_{1}-e_{2}} x^{e_{1}-b} M^{e_{1}-b} \frac{(1+x M)}{1-x} .
\end{aligned}
$$

(iii) $\sum_{n \geq 0}\left(\sum_{T \in \mathcal{T}_{2 n}^{\left(2 e_{1}+2,2 e_{2}+1\right)}} \operatorname{sign}(T)\right) x^{n}=\sum_{a=0}^{e_{2}} \sum_{b=0}^{e_{1}-e_{2}} x^{e_{1}-a-b} M^{e_{1}-a-b+1}$.

For the skew part $\mu=\left(2 e_{1}+1,2 e_{2}+1\right)$, since our approach does not apply in this case, so far we have no solution. See the discussion in the concluding remarks.

Let $\mathcal{R}_{n} \subset \mathcal{T}_{n}$ be the subset of $n$-cell SYTs of all shapes with at most two rows. It is known that the cardinality of $\mathcal{R}_{n}$ is $\left(\begin{array}{c}n \\ \lfloor n / 2\rfloor\end{array}\right)$ [1, Corollary 3.4]. We obtain the following sign-balance results. 
Theorem 1.3. For $n \geq 1$, the following results hold.

(i) $\sum_{T \in \mathcal{R}_{2 n}} \operatorname{sign}(T)=2\left(\begin{array}{c}n-1 \\ \left\lfloor\frac{n-1}{2}\right\rfloor\end{array}\right)$.
(ii) $\sum_{T \in \mathcal{R}_{2 n+1}} \operatorname{sign}(T)=\left(\begin{array}{c}n \\ \lfloor n / 2\rfloor\end{array}\right)$.

\section{Preliminaries}

\subsection{Yamanouchi words}

Given an $n$-cell tableau $T$, we associate $T$ with a word $\zeta(T)=u_{1} \cdots u_{n}$ on the alphabet $\{1,2,3, \ldots\}$, where $\zeta(T)$ is obtained from $T$ by letting the $j$ th letter $u_{j}$ be the row index of the cell of $T$ containing the number $j$. The words $\zeta(T)$ are known as Yamanouchi words. For example,

$$
T=\begin{array}{l|l|l}
\hline 1 & 3 & 6 \\
\hline 2 & 5 & 7 \\
\hline 4 & 8 &
\end{array} \quad \zeta(T)=12132123 .
$$

Note that the $i$ th row of $T$ contains the indices of the letter $i$ in $\zeta(T)$.

The inversion number of $\zeta(T)$, denoted by $\operatorname{inv}(\zeta(T))$, is defined to be the number of pairs $\left(u_{i}, u_{j}\right), 1 \leq i<j \leq n$ such that $u_{i}>u_{j}$.

Lemma 2.1. For any standard Young tableau $T$, we have $\operatorname{sign}(T)=(-1)^{\operatorname{inv}(\zeta(T))}$.

Proof. Let $\sigma(T)=\sigma_{1} \sigma_{2} \cdots \sigma_{n}$ be the permutation obtained from $T$ by reading the entries row by row, and let $\zeta(T)=u_{1} \cdots u_{n}$ be the Yamanouchi word associated with $T$. For each $\sigma_{j}(1 \leq j \leq n)$, say $\sigma_{j}=k$, we observe that the entries to the left of $\sigma_{j}$ and greater than $\sigma_{j}$ in $\sigma(T)$ are in one-to-one correspondence with the entries to the right of $u_{k}$ and less than $u_{k}$ in $\zeta(T)$. Hence $\operatorname{inv}(\zeta(T))=\operatorname{inv}(\sigma(T))$. The assertion follows.

\subsection{Skew standard domino tableaux}

A domino is a pair of cells that share an edge. It is called a horizontal (vertical, respectively) domino if the two cells are in the same row (column, respectively). An $n$-domino skew standard domino tableau $T$ of shape $\lambda / \mu$ is a $2 n$-cell skew standard Young tableau of the same shape in which the two cells with entries $2 i-1$ and $2 i$ form a domino for $1 \leq i \leq n$. If the skew part $\mu$ is empty then $T$ is called a standard domino tableau (SDT). Let $\mathcal{A}_{n}^{\left(\mu_{1}, \mu_{2}\right)} \subset \mathcal{T}_{2 n}^{\left(\mu_{1}, \mu_{2}\right)}$ denote the set of $n$-domino skew SDTs of all shapes with at most three rows and the skew part $\mu=\left(\mu_{1}, \mu_{2}\right)$.

Lemma 2.2. The following results hold. 
(i) For the skew SYTs with an even number of cells, we have

$$
\sum_{T \in \mathcal{T}_{2 n}^{\left(\mu_{1}, \mu_{2}\right)}} \operatorname{sign}(T)=\sum_{T \in \mathcal{A}_{n}^{\left(\mu_{1}, \mu_{2}\right)}} \operatorname{sign}(T) .
$$

(ii) In particular, for the SYTs with an odd number of cells we have

$$
\sum_{T \in \mathcal{T}_{2 n+1}} \operatorname{sign}(T)=\sum_{T \in \mathcal{A}_{n}^{(1,0)}} \operatorname{sign}(T) .
$$

Proof. (i) Given a tableau $T \in \mathcal{T}_{2 n}^{\left(\mu_{1}, \mu_{2}\right)} \backslash \mathcal{A}_{n}^{\left(\mu_{1}, \mu_{2}\right)}$, find the least integer $j$ such that the two cells with entries $2 j-1$ and $2 j$ are not adjacent. Notice that they are in different rows necessarily. Then interchanging the two entries becomes a sign-reversing involution on the tableaux. The assertion follows.

(ii) There is an immediate bijection $T \mapsto T^{\prime}$ of $\mathcal{T}_{2 n+1}$ onto $\mathcal{T}_{2 n}^{(1,0)}$ such that $T^{\prime}$ is obtained from $T$ by removing the entry 1 and subtract the other entries by 1 . By the same argument as in (i), the assertion follows.

We say that a vertical domino in a skew SDT is a $(1,2)$-domino $((2,3)$-domino, respectively) if the two cells are in row 1 and row 2 (row 2 and row 3 , respectively).

Lemma 2.3. For any tableau $T \in \mathcal{A}_{n}^{\left(\mu_{1}, \mu_{2}\right)}$, if $\mu_{1} \mu_{2}$ is odd then every $(2,3)$-domino of $T$ is preceded by an odd number of $(1,2)$-dominoes; otherwise, every $(2,3)$-domino of $T$ is preceded by an even number of $(1,2)$-dominoes.

Proof. Notice that if $\mu_{1}$ is even and $\mu_{2}$ is odd then $T$ contains no vertical dominoes. So, we consider the remaining cases regarding the parities of $\mu_{1}$ and $\mu_{2}$.

Let $\zeta(T)=u_{1} \cdots u_{2 n}$. Suppose $T$ contains a $(2,3)$-domino $U$, say $U=\left(u_{2 j-1}, u_{2 j}\right)$ for some $j$. Then the number of 2's and the number of 3's in the prefix $u_{1} \cdots u_{2 j-2}$ of $\zeta(T)$ have the same (opposite, respectively) parity if $\mu_{2}$ is even (odd, respectively). Note that each $(1,2)$-domino contributes a letter 2 and no letter 3 to the word $\zeta(T)$, and that each $(2,3)$-domino contributes a letter 2 and a letter 3 . Hence if $\mu_{2}$ is even then there are an even number of $(1,2)$-dominoes preceding $U$. Moreover, if $\mu_{2}$ is odd then $\mu_{1}$ is odd and there are an odd number of $(1,2)$-dominoes preceding $U$.

We observe that the sign of a skew SDT in $\mathcal{A}_{n}^{\left(\mu_{1}, \mu_{2}\right)}$ can be determined by the number of vertical dominoes it contains.

Proposition 2.4. For any tableau $T \in \mathcal{A}_{n}^{\left(\mu_{1}, \mu_{2}\right)}$ with $d$ vertical dominoes, the following properties hold. 
(i) If $\mu_{1} \mu_{2}$ is even then we have $\operatorname{sign}(T)=+1$ if $d \equiv 0,1(\bmod 4)$, and $\operatorname{sign}(T)=-1$ if $d \equiv 2,3(\bmod 4)$.

(ii) If $\mu_{1} \mu_{2}$ is odd then we have $\operatorname{sign}(T)=+1$ if $d-2 b \equiv 0,1(\bmod 4)$, and $\operatorname{sign}(T)=-1$ if $d-2 b \equiv 2,3(\bmod 4)$, where $b$ is the number of $(2,3)$-dominoes in $T$.

Proof. Let $\zeta(T)=u_{1} \cdots u_{2 n}$. For any two dominoes $U, V$, say $U=\left(u_{2 i-1}, u_{2 i}\right)$ and $V=\left(u_{2 j-1}, u_{2 j}\right), i<j$, we say that the number of inversions contributed by the ordered pair $(U, V)$, denoted by $\operatorname{inv}(U, V)$, is the number of inversions of the subsequence $u_{2 i-1} u_{2 i} u_{2 j-1} u_{2 j}$. Then $\operatorname{inv}(\zeta(T))$ is the total number of inversions of all pairs of dominoes.

We observe that if at least one of $U, V$ is a horizontal domino then $\operatorname{inv}(U, V)$ is even. Moreover, if both of $U, V$ are vertical dominoes then we have

$$
\operatorname{inv}(U, V)= \begin{cases}0 & \text { if } U=(1,2) \text { and } V=(2,3) \\ 1 & \text { if } U=V=(1,2) \text { or } U=V=(2,3) \\ 3 & \text { if } U=(2,3) \text { and } V=(1,2)\end{cases}
$$

There are totally $\left(\begin{array}{l}d \\ 2\end{array}\right)$ pairs of vertical dominoes in $T$.

(i) If at least one of $\mu_{1}, \mu_{2}$ is even then by Lemma 2.3 , there are an even number of pairs $(U, V)$ such that $U=(1,2)$ and $V=(2,3)$, which contribute no inversions. Since each of the remaining ordered pairs $(U, V)$ contributes an odd number of inversions, $\operatorname{inv}(\zeta(T))$ has the same parity of $d(d-1) / 2$. By Lemma 2.1, the assertion (i) follows.

(ii) Otherwise, both of $\mu_{1}, \mu_{2}$ are odd. Let $b$ be the number of $(2,3)$-dominoes in $T$. Suppose $d$ is odd. We observe that the number $b$ is always even if $d$ is odd. Then there are an even number of pairs $(U, V)$ such that $U=(1,2)$ and $V=(2,3)$, and hence $\operatorname{inv}(\zeta(T))$ has the same parity of $d(d-1) / 2$.

Suppose $d$ is even. Notice that the number of pairs $(U, V)$ such that $U=(1,2)$ and $V=(2,3)$ has the same parity of $b$, and that $\operatorname{inv}(\zeta(T))$ has the same parity of $d(d-1) / 2-b$. Consider the following two cases.

- $d \equiv 0(\bmod 4)$. We observe that $d-2 b \equiv 0(\bmod 4)$ if $b$ is even, and $d-2 b \equiv 2$ $(\bmod 4)$ if $b$ is odd. Since the total number $d(d-1) / 2$ of pairs $(U, V)$ is even, we have $\operatorname{sign}(T)=+1$ if $d-2 b \equiv 0(\bmod 4)$, and $\operatorname{sign}(T)=-1$ if $d-2 b \equiv 2(\bmod 4)$.

- $d \equiv 2(\bmod 4)$. We observe that $d-2 b \equiv 0(\bmod 4)$ if $b$ is odd, and $d-2 b \equiv 2$ $(\bmod 4)$ if $b$ is even. Since the total number $d(d-1) / 2$ of pairs $(U, V)$ is odd, we have $\operatorname{sign}(T)=+1$ if $d-2 b \equiv 0(\bmod 4)$, and $\operatorname{sign}(T)=-1$ if $d-2 b \equiv 2(\bmod 4)$.

The assertion (ii) follows. 


\subsection{Motzkin paths}

A Motzkin path of length $n$ is a lattice path from the origin to the point $(n, 0)$ staying weakly above the $x$-axis, using the up step $(1,1)$, down step $(1,-1)$, and level step $(1,0)$. Let U, D and L denote an up step, a down step, and a level step, respectively. A partial Motzkin path of length $n$ is a lattice path from the origin to the line $x=n$ staying weakly above the $x$-axis, using the set $\{\mathrm{U}, \mathrm{D}, \mathrm{L}\}$ of steps. Sometimes a segment of a partial Motzkin path is itself a Motzkin path raised to a certain level. For convenience, the horizontal line where a Motzkin path starts, stays weakly above and ends is called the base line of the path. Let $\mathcal{M}_{n}$ be the set of partial Motzkin paths of length $n$. Our approach relies on the following bijective results.

Theorem 2.5. (Cheng-Eu-Fu-Lee [2]) The following results hold.

(i) There is a bijection $\Phi: \mathcal{A}_{n} \rightarrow \mathcal{M}_{n}$ such that a tableau $T \in \mathcal{A}_{n}$ with $d$ vertical dominoes is carried to a path $\Phi(T) \in \mathcal{M}_{n}$ from $(0,0)$ to $(n, d)$ for $0 \leq d \leq n$.

(ii) There is a bijection $\Phi: \mathcal{A}_{n}^{(1,0)} \rightarrow \mathcal{M}_{n}$ such that a tableau $T \in \mathcal{A}_{n}^{(1,0)}$ with $d$ vertical dominoes is carried to a path $\Phi(T) \in \mathcal{M}_{n}$ from $(0,0)$ to $(n, d)$ for $0 \leq d \leq n$.

We refer the readers to [2, Section 2] for the construction of the bijection.

\section{Proof of Theorem 1.1}

We shall establish a sign-reversing involution $\Gamma: \mathcal{A}_{n} \rightarrow \mathcal{A}_{n}$, making use of the bijective result in Theorem 2.5(i).

First, we describe the set $\mathcal{F}_{n} \subset \mathcal{A}_{n}$ of fixed points of the map $\Gamma$. The set $\mathcal{F}_{n}$ consists of the tableaux $T \in \mathcal{A}_{n}$ such that the associated partial Motzkin Dyck path $\Phi(T)$ is in either one of the following forms:

(F1) $\Phi(T)=\mathrm{L}^{n}$, consisting of $n$ level steps.

(F2) $\Phi(T)=\mathrm{L}^{k} \mathrm{U} \pi$ for some $k, 0 \leq k \leq n-1$, where $\mathrm{U}$ is an up step, which goes from the $x$-axis to the line $y=1$, and $\pi$ is a Motzkin path of length $n-k-1$ (with the base line $y=1)$.

Notice that $\operatorname{sign}(T)=+1$ since the former (latter, respectively) tableau $T$ contains zero (one, respectively) vertical domino. Moreover, by the conditions (F1) and (F2), the number of fixed points of the map $\Gamma$ can be determined immediately.

Lemma 3.1. For $n \geq 1$, we have

$$
\left|\mathcal{F}_{n}\right|=1+m_{0}+m_{1}+\cdots+m_{n-1} .
$$


Now, we describe the construction of the map $\Gamma$.

Algorithm A. Given a tableau $T \in \mathcal{A}_{n} \backslash \mathcal{F}_{n}$ with $d$ vertical dominoes for some $d \geq 0$, construct the associated partial Motzkin path $\Phi(T)$. There is a standard factorization of the path $\Phi(T)$ into Motzkin paths as

$$
\Phi(T)=\alpha_{0} \mathrm{U}_{1} \alpha_{1} \cdots \mathrm{U}_{d} \alpha_{d}
$$

where $\mathrm{U}_{j}$ is the last up step going from the line $y=j-1$ to the line $y=j, 1 \leq j \leq d$, and each segment $\alpha_{i}$ is a Motzkin path of a certain length (possibly empty) with the base line $y=i, 0 \leq i \leq d$. We create a path $\beta$ from $\Phi(T)$ according to the following cases.

Case 1. The segment $\alpha_{0}$ contains up steps and down steps. Then find the first down step going from the line $y=1$ to the $x$-axis, say $z_{a}$. Then the path $\beta$ is obtained from $\Phi(T)$ by replacing $z_{a}$ by an up step, which goes from the line $y=1$ to the line $y=2$.

Case 2. The segment $\alpha_{0}$ consists of level steps (possibly empty). Since the tableau $T$ is not a fixed point, by (F1) and (F2), we have $d \geq 2$. Then find the last up step going from the line $y=1$ to the line $y=2$, say $z_{b}$. Then the path $\beta$ is obtained from $\Phi(T)$ by replacing $z_{b}$ by a down step, which goes from the line $y=1$ to the $x$-axis.

Then the requested tableau $\Gamma(T)$ is defined to be the tableau associated with $\beta$, i.e., $\Gamma(T)=\Phi^{-1}(\beta)$. By Proposition 2.4, we observe that $T$ and $\Gamma(T)$ have the opposite signs since the end point of $\beta$ is at height either $d+2$ or $d-2$.

Example 3.2. Given the tableau $T \in \mathcal{A}_{15}$ shown in Figure 3.1(a), the partial Motzkin path $\Phi(T)$ is shown in Figure 3.1(b). The path $\beta$ constructed from $\Phi(T)$ by Algorithm A is shown in Figure $3.2(\mathrm{~b})$, and the corresponding tableau $\Gamma(T)=\Phi^{-1}(\beta)$ is shown in Figure 3.2 (a).

\begin{tabular}{|c|c|c|c|c|c|c|}
\hline 1 & 4 & 5 & \multirow{2}{*}{8} & 11 & 14 & 15 \\
\hline 2 & 6 & 7 & & 12 & & \\
\hline 3 & 9 & 10 & & & & \\
\hline
\end{tabular}

(a)

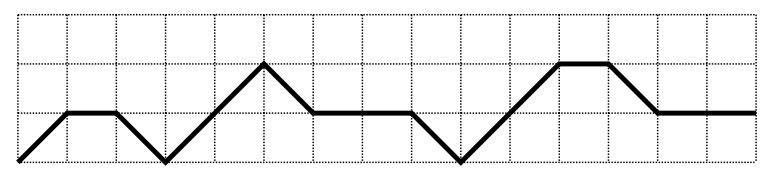

(b)

Figure 3.1: A tableau in $\mathcal{A}_{15}$ and the associated path under the map $\Phi$. 


\begin{tabular}{|c|c|c|c|c|c|c|c|}
\hline \multirow{2}{*}{1} & \multirow{2}{*}{2} & 4 & 5 & \multirow{2}{*}{8} & 11 & 14 & \multirow[t]{3}{*}{15} \\
\hline & & 6 & 7 & & 12 & & \\
\hline & 3 & 9 & 10 & & & & \\
\hline
\end{tabular}

(a)

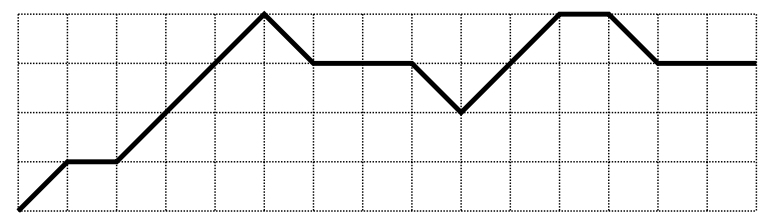

(b)

Figure 3.2: The corresponding tableau of the tableau in Figure 3.1 under the map $\Gamma$.

Proof of Theorem 1.1. (i) By Lemmas 2.2 and 3.1, we have

$$
\sum_{T \in \mathcal{T}_{2 n}} \operatorname{sign}(T)=\sum_{T \in \mathcal{A}_{n}} \operatorname{sign}(T)=\left|\mathcal{F}_{n}\right|=1+m_{0}+m_{1}+\cdots+m_{n-1} .
$$

The assertion (ii) can be proved in the same manner, by establishing a sign-reversing involution on the set $\mathcal{A}_{n}^{(1,0)}$ by the construction in Algorithm A, making use of the bijective result in Theorem 2.5(ii).

\section{Proof of Theorem 1.2}

In this section, we shall prove Theorem 1.2 by an extension of the approach used in the previous section.

\subsection{On tableaux with the skew part $\mu=\left(2 e_{1}, 2 e_{2}\right)$}

We describe a classification of the tableaux in $\mathcal{A}_{n}^{\left(2 e_{1}, 2 e_{2}\right)}$ according to the path converted from the skew part $\mu=\left(2 e_{1}, 2 e_{2}\right)$, given by Cheng et al. [2, Scetion 3].

Given a skew $\operatorname{SDT} T \in \mathcal{A}_{n}^{\left(2 e_{1}, 2 e_{2}\right)}$, create a $\operatorname{SDT} \bar{T} \in \mathcal{A}_{n+e_{1}+e_{2}}$ by tiling the skew part with horizontal dominoes such that the dominoes in the first (second, respectively) row are labeled by $\left\{1,3, \ldots, 2 e_{2}-1\right\} \cup\left\{2 e_{2}+1,2 e_{2}+2, \ldots, e_{1}+e_{2}\right\}\left(\left\{2,4, \ldots, 2 e_{2}\right\}\right.$, respectively). The original labels in $T$ are then shifted from $\{1, \ldots, n\}$ to $\left\{e_{1}+e_{2}+1, \ldots, e_{1}+e_{2}+n\right\}$. Construct the corresponding path $\Phi(\bar{T}) \in \mathcal{M}_{n+e_{1}+e_{2}}$ and factorize it into two parts with lengths $e_{1}+e_{2}$ and $n$, respectively, as

$$
\Phi(\bar{T})=\nu \beta
$$

Note that the initial segment $\nu$ is converted from the domino-tiling of the skew part $\mu=\left(2 e_{1}, 2 e_{2}\right)$. We classify the tableaux according to the form of $\nu$ as follows. 
The set $\mathcal{A}_{n}^{\left(2 e_{1}, 2 e_{2}\right)}$ can be partitioned into subsets $\mathcal{A}_{n ; a, b}^{\left(2 e_{1}, 2 e_{2}\right)}$ for $0 \leq a \leq e_{2}$ and $0 \leq b \leq$ $e_{1}-e_{2}$, where $\mathcal{A}_{n ; a, b}^{\left(2 e_{1}, 2 e_{2}\right)}$ consists of the tableaux $T$ such that the segment $\nu$ of $\Phi(\bar{T})$ is of the form

$$
\nu=(\mathrm{UD})^{a}(\mathrm{UL})^{e_{2}-a} \mathrm{~L}^{b} \mathrm{U}^{e_{1}-e_{2}-b} .
$$

Note that the path $\nu$ goes from the origin to the point $\left(e_{1}+e_{2}, e_{1}-a-b\right)$, and $\beta$ goes from the point $\left(e_{1}+e_{2}, e_{1}-a-b\right)$ to the line $x=n+e_{1}+e_{2}$. In particular, the subsets with $a>0$ and $b>0$ in the classification have the following property.

Lemma 4.1. 2, Lemma 3.1] For $a>0$ and $b>0$, every tableau $T$ in the set $\mathcal{A}_{n ; a, b}^{\left(2 e_{1}, 2 e_{2}\right)}$ contains no vertical domino.

We shall establish a sign-reversing involution $\Gamma$ on each set $\mathcal{A}_{n ; a, b}^{\left(2 e_{1}, 2 e_{2}\right)}$. Consider the factorization $\Phi(\bar{T})=\nu \beta$ in 4.1 . For convenience, we remove the segment $\nu$ and view the segment $\beta$ as a lattice path from the point $\left(0, e_{1}-a-b\right)$ to the line $x=n$. Note that the path $\beta$ touches the $x$-axis necessarily [2, Section 3]. With respect to the first down step returning to the $x$-axis, we further factorize $\beta$ into two parts as

$$
\beta=\beta_{1} \beta_{2}
$$

where $\beta_{1}$ goes from the point $\left(0, e_{1}-a-b\right)$ to the $x$-axis (for the first time), say at $(t, 0)$ for some $t\left(e_{1}-a-b \leq t \leq n\right)$, and $\beta_{2}$ is a partial Motzkin path from the point $(t, 0)$ to the line $x=n$.

We describe the set $\mathcal{F}_{n ; a, b}^{\left(2 e_{1}, 2 e_{2}\right)} \subseteq \mathcal{A}_{n ; a, b}^{\left(2 e_{1}, 2 e_{2}\right)}$ of fixed points of the map $\Gamma$ in the following two cases.

Case 1. If $a>0$ and $b>0$ then $\mathcal{F}_{n ; a, b}^{\left(2 e_{1}, 2 e_{2}\right)}=\mathcal{A}_{n ; a, b}^{\left(2 e_{1}, 2 e_{2}\right)}$, i.e., $\Gamma(T)=T$ for any $T \in \mathcal{A}_{n ; a, b}^{\left(2 e_{1}, 2 e_{2}\right)}$. In this case, the end point of the segment $\beta_{2}$ is $(n, 0)$ since the tableau $T$ contains no vertical domino. Hence $\beta_{2}$ is a Motzkin path.

Case 2. If $a=0$ or $b=0$ then the set $\mathcal{F}_{n ; a, b}^{\left(2 e_{1}, 2 e_{2}\right)}$ consists of the skew SDTs $T$ such that the segment $\beta_{2}$ is in either one of the following two forms:

$\left(\mathrm{F} 1^{\prime}\right) \beta_{2}=\mathrm{L}^{n-t}$.

(F2') $\beta_{2}=\mathrm{L}^{k} \mathrm{U} \pi$ for some $k, 0 \leq k \leq n-t-1$, where $\mathrm{U}$ is an up step going from the $x$-axis to the line $y=1$ and $\pi$ is a Motzkin path of length $n-t-k-1$ (with the base line $y=1)$.

The enumeration of the fixed points can be expressed in terms of Motzkin number. Recall that by Proposition 2.4, the fixed points are positive since the tableaux in the former (latter, respectively) case contain no (one, respectively) vertical domino. 
Lemma 4.2. The following results hold.

(i) If $a>0$ and $b>0$ then we have

$$
\sum_{n \geq 0}\left|\mathcal{F}_{n ; a, b}^{\left(2 e_{1}, 2 e_{2}\right)}\right| x^{n}=x^{e_{1}-a-b} M^{e_{1}-a-b+1} .
$$

(ii) If $a=0$ or $b=0$ then we have

$$
\sum_{n \geq 0}\left|\mathcal{F}_{n ; a, b}^{\left(2 e_{1}, 2 e_{2}\right)}\right| x^{n}=x^{e_{1}-a-b} M^{e_{1}-a-b} \frac{(1+x M)}{1-x} .
$$

Proof. We enumerate the tableaux $T \in \mathcal{F}_{n ; a, b}^{\left(2 e_{1}, 2 e_{2}\right)}$ in terms of the segment $\beta$ of the associated path $\Phi(\bar{T})$.

By the factorization $\beta=\beta_{1} \beta_{2}$ in (4.3), there is a standard factorization of former part $\beta_{1}$ into Motzkin paths as $\beta_{1}=\pi_{e_{1}-a-b} \mathrm{D}_{e_{1}-a-b} \cdots \pi_{1} \mathrm{D}_{1}$, where $\mathrm{D}_{i}$ is the last down step going from the line $y=i$ to the line $y=i-1$ for $1 \leq i \leq e_{1}-a-b$, and $\pi_{i}$ is a Motzkin path with the base line $y=i$.

(i) If $a>0$ and $b>0$ then the latter part $\beta_{2}$ is also a Motzkin path. We observe that

$$
\left|\mathcal{F}_{n ; a, b}^{\left(2 e_{1}, 2 e_{2}\right)}\right|=\sum_{\substack{k_{0}+k_{1}+\cdots+k_{e_{1}-a-b} \\=n-e_{1}+a+b}} m_{k_{0}} m_{k_{1}} \cdots m_{k_{e_{1}-a-b}} .
$$

(ii) If $a=0$ or $b=0$ then $\beta_{2}$ is in either one of the forms (F1') and (F2'). We observe that

$$
\left|\mathcal{F}_{n ; a, b}^{\left(2 e_{1}, 2 e_{2}\right)}\right|=\sum_{t=e_{1}-a-b}^{n}\left(\sum_{\substack{k_{1}+\cdots+k_{e_{1}-a-b} \\=t-e_{1}+a+b}} m_{k_{1}} \cdots m_{k_{e_{1}-a-b}}\left(1+m_{0}+m_{1}+\cdots+m_{n-t-1}\right)\right) .
$$

The assertions follow from multiplying the equation by $x^{n}$ and summing over $n \geq 0$.

Next, we construct the map $\Gamma$ on the set $\mathcal{A}_{n ; a, b}^{\left(2 e_{1}, 2 e_{2}\right)}$ with $a=0$ or $b=0$, by the same operations as in Algorithm A.

Algorithm B. Given a tableau $T \in \mathcal{A}_{n ; a, b}^{\left(2 e_{1}, 2 e_{2}\right)} \backslash \mathcal{F}_{n ; a, b}^{\left(2 e_{1}, 2 e_{2}\right)}$ with $d$ vertical dominoes for some $d \geq 0$, construct the associated path $\Phi(\bar{T})=\nu \beta$. By the factorization $\beta=\beta_{1} \beta_{2}$ in (4.3), the latter part $\beta_{2}$ goes from somewhere in the $x$-axis to the point $(n, d)$. We factorize $\beta_{2}$ as

$$
\beta_{2}=\alpha_{0} \mathrm{U}_{1} \alpha_{1} \cdots \mathrm{U}_{d} \alpha_{d}
$$

where $\mathrm{U}_{j}$ is the last up step going from the line $y=j-1$ to the line $y=j, 1 \leq j \leq d$, and each segment $\alpha_{i}$ is a Motzkin path of a certain length (possibly empty) with the base line $y=i, 0 \leq i \leq d$. We create a path $\beta^{\prime}$ from $\beta$ according to the following cases. 
Case 1. The segment $\alpha_{0}$ contains up steps and down steps. Then find the first down step going from the line $y=1$ to the $x$-axis, say $z_{a}$. Then the path $\beta^{\prime}$ is obtained from $\beta$ by replacing $z_{a}$ by an up step, which goes from the line $y=1$ to the line $y=2$.

Case 2. The segment $\alpha_{0}$ consists of level steps (possibly empty). Then $d \geq 2$ and find the last up step going from the line $y=1$ to the line $y=2$, say $z_{b}$. Then the path $\beta^{\prime}$ is obtained from $\beta$ by replacing $z_{b}$ by a down step, which goes from the line $y=1$ to the $x$-axis.

To find the corresponding tableau $\Gamma(T)$, we construction a SDT from the path $\nu \beta^{\prime}$ by $\Phi^{-1}$, i.e., $\bar{T}^{\prime}=\Phi^{-1}\left(\nu \beta^{\prime}\right)$. Then the requested tableau $\Gamma(T)$ is obtained from $\bar{T}^{\prime}$ by removing the skew part. By Proposition 2.4. we observe that $T$ and $\Gamma(T)$ have the opposite signs since the end point of $\beta^{\prime}$ is at height either $d+2$ or $d-2$.

Now, we prove Theorem 1.2 (i).

Proof of Theorem 1.2(i). By Lemmas 2.2 and 4.2 , we have

$$
\begin{aligned}
& \sum_{n \geq 0}\left(\sum_{T \in \mathcal{T}_{2 n}^{\left(2 e_{1}, 2 e_{2}\right)}} \operatorname{sign}(T)\right) x^{n} \\
= & \sum_{n \geq 0}\left(\sum_{T \in \mathcal{A}_{n}^{\left(2 e_{1}, 2 e_{2}\right)}} \operatorname{sign}(T)\right) x^{n}=\sum_{a=0}^{e_{2}} \sum_{b=0}^{e_{1}-e_{2}} \sum_{n \geq 0}\left(\sum_{T \in \mathcal{A}_{n ; a, b}^{\left(2 e_{1}, 2 e_{2}\right)}} \operatorname{sign}(T)\right) x^{n} \\
= & \sum_{a=1}^{e_{2}} \sum_{b=1}^{e_{1}-e_{2}} \sum_{n \geq 0}\left|\mathcal{F}_{n ; a, b}^{\left(2 e_{1}, 2 e_{2}\right)}\right| x^{n}+\sum_{a=0}^{e_{2}} \sum_{b=0}^{e_{1}-e_{2}} \chi(a b=0) \sum_{n \geq 0}\left|\mathcal{F}_{n ; a, b}^{\left(2 e_{1}, 2 e_{2}\right)}\right| x^{n} \\
= & \sum_{a=1}^{e_{2}} \sum_{b=1}^{e_{1}-e_{2}} x^{e_{1}-a-b} M^{e_{1}-a-b+1}+\sum_{a=0}^{e_{2}} \sum_{b=0}^{e_{1}-e_{2}} \chi(a b=0) x^{e_{1}-a-b} M^{e_{1}-a-b} \frac{(1+x M)}{1-x} .
\end{aligned}
$$

4.2. On tableaux with the skew part $\mu=\left(2 e_{1}+1,2 e_{2}\right)$

To prove Theorem 1.2(ii), we shall establish a sign-reversing involution on the set $\mathcal{A}_{n}^{\left(2 e_{1}+1,2 e_{2}\right)}$ in the same manner as above.

We make use of a classification of the tableaux given by Cheng et al. [2, Section 5]. Given a tableau $T \in \mathcal{A}_{n}^{\left(2 e_{1}+1,2 e_{2}\right)}$, create a skew SDT $\bar{T} \in \mathcal{A}_{n+e_{1}+e_{2}}^{(1,0)}$ by tiling the shape $\left(2 e_{1}+1,2 e_{2}\right) /(1,0)$ with horizontal dominoes such that the dominoes in the first (second, respectively) row are labeled by $\left\{1,3, \ldots, 2 e_{2}-1\right\} \cup\left\{2 e_{2}+1,2 e_{2}+2, \ldots, e_{1}+e_{2}\right\}$ $\left(\left\{2,4, \ldots, 2 e_{2}\right\}\right.$, respectively). The original labels in $T$ are then shifted from $\{1, \ldots, n\}$ to $\left\{e_{1}+e_{2}+1, \ldots, e_{1}+e_{2}+n\right\}$. Construct the corresponding path $\Phi(\bar{T}) \in \mathcal{M}_{n+e_{1}+e_{2}}$ and 
factorize it into two parts as $\Phi(\bar{T})=\nu \beta$, where $\nu$ is converted from the domino-tiling of the shape $\left(2 e_{1}+1,2 e_{2}\right) /(1,0)$. The tableaux $T$ in $\mathcal{A}_{n}^{\left(2 e_{1}+1,2 e_{2}\right)}$ are classified as follows.

The set $\mathcal{A}_{n}^{\left(2 e_{1}+1,2 e_{2}\right)}$ can be partitioned into subsets $\mathcal{A}_{n ; a, b}^{\left(2 e_{1}+1,2 e_{2}\right)}$ for $0 \leq a \leq e_{2}$ and $0 \leq b \leq e_{1}-e_{2}$, where $\mathcal{A}_{n ; a, b}^{\left(2 e_{1}+1,2 e_{2}\right)}$ consists of the tableaux $T$ such that the segment $\nu$ of $\Phi(\bar{T})$ is of the form

$$
\nu=(\mathrm{UD})^{a}(\mathrm{UL})^{e_{2}-a} \mathrm{~L}^{b} \mathrm{U}^{e_{1}-e_{2}-b} .
$$

In the classification, the subsets with $a>0$ have the following property.

Lemma 4.3. [2, Lemma 5.1] For a $>0$, every tableau $T$ in the set $\mathcal{A}_{n ; a, b}^{\left(2 e_{1}+1,2 e_{2}\right)}$ contains no vertical domino.

We describe the set $\mathcal{F}_{n ; a, b}^{\left(2 e_{1}+1,2 e_{2}\right)} \subset \mathcal{A}_{n ; a, b}^{\left(2 e_{1}+1,2 e_{2}\right)}$ of fixed points of the map $\Gamma$. For a tableau $T \in \mathcal{A}_{n ; a, b}^{\left(2 e_{1}+1,2 e_{2}\right)}$, consider the factorization of the path $\Phi(\bar{T})=\nu \beta$ in 4.1 . With the segment $\nu$ removed, we further factorize the segment $\beta$ into two parts $\beta_{1} \beta_{2}$ as in 4.3 . Consider the following two cases.

Case 1. If $a>0$ then $\mathcal{F}_{n ; a, b}^{\left(2 e_{1}+1,2 e_{2}\right)}=\mathcal{A}_{n ; a, b}^{\left(2 e_{1}+1,2 e_{2}\right)}$. In this case, the segment $\beta_{2}$ is a Motzkin path.

Case 2. If $a=0$ then the set $\mathcal{F}_{n ; a, b}^{\left(2 e_{1}+1,2 e_{2}\right)}$ consists of the skew SDTs $T$ such that the segment $\beta_{2}$ is in either of the two forms given in (F1') and (F2').

By the same argument as in the proof of Lemma 4.2, we obtain the following enumeration of the fixed points.

Lemma 4.4. The following results hold.

(i) If $a>0$ then we have

$$
\sum_{n \geq 0}\left|\mathcal{F}_{n ; a, b}^{\left(2 e_{1}+1,2 e_{2}\right)}\right| x^{n}=x^{e_{1}-a-b} M^{e_{1}-a-b} .
$$

(ii) If $a=0$ then we have

$$
\sum_{n \geq 0}\left|\mathcal{F}_{n ; a, b}^{\left(2 e_{1}+1,2 e_{2}\right)}\right| x^{n}=x^{e_{1}-b} M^{e_{1}-b} \frac{(1+x M)}{1-x} .
$$

For the case $a=0$, the requested map $\Gamma: \mathcal{A}_{n ; a, b}^{\left(2 e_{1}+1,2 e_{2}\right)} \rightarrow \mathcal{A}_{n ; a, b}^{\left(2 e_{1}+1,2 e_{2}\right)}$ can be established by the construction described in Algorithm B. Now, we prove Theorem 1.2(ii).

Proof of Theorem 1.2(ii). By Lemmas 2.2 and 4.2 , we have

$$
\sum_{n \geq 0}\left(\sum_{T \in \mathcal{T}_{2 n}^{\left(2 e_{1}+1,2 e_{2}\right)}} \operatorname{sign}(T)\right) x^{n}
$$




$$
\begin{aligned}
& =\sum_{n \geq 0}\left(\sum_{T \in \mathcal{A}_{n}^{\left(2 e_{1}+1,2 e_{2}\right)}} \operatorname{sign}(T)\right) x^{n}=\sum_{a=0}^{e_{2}} \sum_{b=0}^{e_{1}-e_{2}} \sum_{n \geq 0}\left(\sum_{T \in \mathcal{A}_{n ; a, b}^{\left(2 e_{1}+1,2 e_{2}\right)}} \operatorname{sign}(T)\right) x^{n} \\
& =\sum_{a=1}^{e_{2}} \sum_{b=0}^{e_{1}-e_{2}} \sum_{n \geq 0}\left|\mathcal{F}_{n ; a, b}^{\left(2 e_{1}+1,2 e_{2}\right)}\right| x^{n}+\sum_{b=0}^{e_{1}-e_{2}} \sum_{n \geq 0}\left|\mathcal{F}_{n ; 0, b}^{\left(2 e_{1}+1,2 e_{2}\right)}\right| x^{n} \\
& =\sum_{a=1}^{e_{2}} \sum_{b=0}^{e_{1}-e_{2}} x^{e_{1}-a-b} M^{e_{1}-a-b+1}+\sum_{b=0}^{e_{1}-e_{2}} x^{e_{1}-b} M^{e_{1}-b} \frac{(1+x M)}{1-x} .
\end{aligned}
$$

\subsection{On tableaux with the skew part $\mu=\left(2 e_{1}+2,2 e_{2}+1\right)$}

Notice that every tableau in $\mathcal{A}_{n}^{\left(2 e_{1}+2,2 e_{2}+1\right)}$ contains no vertical domino. Hence $\operatorname{sign}(T)=1$ for all $T \in \mathcal{A}_{n}^{\left(2 e_{1}+2,2 e_{2}+1\right)}$. Let $\mathcal{H}_{n}^{\left(2 e_{1}, 2 e_{2}\right)} \subset \mathcal{A}_{n}^{\left(2 e_{1}, 2 e_{2}\right)}$ be the subset of tableaux containing no vertical domino. We observe that there is an immediate bijection $T \mapsto T^{\prime}$ of $\mathcal{A}_{n}^{\left(2 e_{1}+2,2 e_{2}+1\right)}$ onto $\mathcal{H}_{n}^{\left(2 e_{1}, 2 e_{2}\right)}$ such that $T^{\prime}$ is obtained from $T$ by shifting the first (second, respectively) row to the left by two cells (one cell, respectively). For the sign-balance of $\mathcal{T}_{2 n}^{\left(2 e_{1}+2,2 e_{2}+1\right)}$, we shall enumerate the tableaux in $\mathcal{H}_{n}^{\left(2 e_{1}, 2 e_{2}\right)}$, under the classification in (4.2).

For $a \geq 0$ and $b \geq 0$, let $\mathcal{H}_{n ; a, b}^{\left(2 e_{1}, 2 e_{2}\right)}=\mathcal{H}_{n}^{\left(2 e_{1}, 2 e_{2}\right)} \cap \mathcal{A}_{n ; a, b}^{\left(2 e_{1}, 2 e_{2}\right)}$. Given a tableau $T \in$ $\mathcal{H}_{n ; a, b}^{\left(2 e_{1}, 2 e_{2}\right)}$, consider the factorization of the associated path $\Phi(\bar{T})=\nu \beta$ in 4.1 . Since $T$ contains no vertical domino, $\operatorname{sign}(T)=+1$ and the segment $\beta$ can be factorized as

$$
\beta=\pi_{e_{1}-a-b} \mathrm{D}_{e_{1}-a-b} \cdots \pi_{1} \mathrm{D}_{1} \pi_{0},
$$

where $\mathrm{D}_{i}$ is the last down step going from the line $y=i$ to the line $y=i-1$ for $1 \leq i \leq e_{1}-a-b$, and $\pi_{i}$ is a Motzkin path with the base line $y=i$ for $0 \leq i \leq e_{1}-a-b$. By the same argument as in the proof of Lemma 4.2, we have

$$
\sum_{n \geq 0}\left|\mathcal{H}_{n ; a, b}^{\left(2 e_{1}, 2 e_{2}\right)}\right| x^{n}=x^{e_{1}-a-b} M^{e_{1}-a-b+1} .
$$

Hence

$$
\begin{aligned}
& \sum_{n \geq 0}\left(\sum_{T \in \mathcal{T}_{2 n}^{\left(2 e_{1}+2,2 e_{2}+1\right)}} \operatorname{sign}(T)\right) x^{n}=\sum_{n \geq 0}\left(\sum_{T \in \mathcal{A}_{n}^{\left(2 e_{1}+2,2 e_{2}+1\right)}} \operatorname{sign}(T)\right) x^{n} \\
= & \sum_{a=0}^{e_{2}} \sum_{b=0}^{e_{1}-e_{2}} \sum_{n \geq 0}\left(\sum_{\substack{\left(2 e_{1}, 2 e_{2}\right) \\
n \in a, b}} \operatorname{sign}(T)\right) x^{n}=\sum_{a=0}^{e_{2}} \sum_{b=0}^{e_{1}-e_{2}} x^{e_{1}-a-b} M^{e_{1}-a-b+1} .
\end{aligned}
$$

The proof of Theorem 1.2 is completed. 
We remark that Cheng et al. gave an explicit formula of $\left|\mathcal{A}_{n}^{\left(2 e_{1}+2,2 e_{2}+1\right)}\right|$ as a linear combination of Motzkin numbers, which involves the Chebyshev polynomials of the second kind [2, Corollary 1.4].

\section{Proof of Theorem 1.3}

Let $\mathcal{B}_{n} \subset \mathcal{A}_{n}$ be the subset of $n$-domino SDTs with at most two rows. First, we describe the structure of images when the map $\Phi: \mathcal{A}_{n} \rightarrow \mathcal{M}_{n}$ restricted to the set $\mathcal{B}_{n}$.

A Dyck path of semi-length $n$ is a Motzkin path of length $2 n$ with no level steps. A dispersed Dyck path of length $n$ is a Motzkin path of length $n$ with no level step above the $x$-axis, which is enumerated by the central binomial coefficients [9, A001405].

Lemma 5.1. The number of dispersed Dyck paths of length $n$ is $\left(\begin{array}{c}n \\ \lfloor n / 2\rfloor\end{array}\right)$.

We have the following observations about the images of the map $\Phi$ restricted to $\mathcal{B}_{n}$.

Proposition 5.2. For a tableau $T \in \mathcal{B}_{n}$, the following properties hold.

(i) If $T$ contains no vertical domino then $\Phi(T)$ is a dispersed Dyck path of length $n$.

(ii) If $T$ contains $d$ vertical dominoes then $\Phi(T)$ can be factorized as

$$
\Phi(T)=\alpha_{0} \mathrm{U}_{1} \cdots \alpha_{d} \mathrm{U}_{d} \pi
$$

where $\mathrm{U}_{j}$ is the last up step going from the line $y=j-1$ to the line $y=j$ for $1 \leq j \leq d, \alpha_{i}$ is a Dyck path (possibly empty) for $0 \leq i \leq d-1$, and $\pi$ is a dispersed Dyck path (possibly empty).

Example 5.3. For the tableau $T \in \mathcal{B}_{15}$ shown in Figure 5.1(a), we have the tiling word $x_{1} x_{2} \cdots x_{15}=V 121122 V 12 V 1121$. The corresponding path $\Phi(T)$ is shown in Figure $5.1(\mathrm{~b})$.

\begin{tabular}{|c|c|c|c|c|c|c|c|c|c|}
\hline \multirow{2}{*}{1} & 2 & 4 & 5 & \multirow{2}{*}{8} & 9 & \multirow{2}{*}{11} & 12 & 13 & 15 \\
\hline & 3 & 6 & 7 & & 10 & & 14 & & \\
\hline
\end{tabular}

(a)

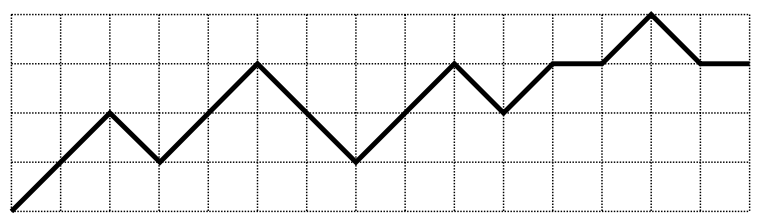

(b)

Figure 5.1: A SDT and the associated path under the map $\Phi$. 
To prove Theorem $1.3(\mathrm{i})$, we shall establish a sign-reversing involution $\Lambda: \mathcal{B}_{n} \rightarrow \mathcal{B}_{n}$ in terms of dispersed Dyck paths.

We describe the set $\mathcal{G}_{n} \subset \mathcal{B}_{n}$ of fixed points of the map $\Lambda$. The set $\mathcal{G}_{n}$ consists of the SDTs $T$ such that the associated path $\Phi(T)$ is in either one of the following forms:

(G1) $\Phi(T)=\mathrm{L} \pi$, where $\pi$ is a dispersed Dyck path of length $n-1$.

(G2) $\Phi(T)=\mathrm{U} \pi$, where $\pi$ is is a dispersed Dyck path of length $n-1$ (with the base line $y=1)$.

By Lemma 5.1 and the conditions (G1) and (G2), the number of fixed points of the map $\Lambda$ is determined.

Lemma 5.4. We have

$$
\left|\mathcal{G}_{n}\right|=2\left(\begin{array}{l}
n-1 \\
\left\lfloor\frac{n-1}{2}\right\rfloor
\end{array}\right) .
$$

Now, we construct the map $\Lambda$ on the set $\mathcal{B}_{n}$.

Algorithm C. Given a tableau $T \in \mathcal{B}_{n} \backslash \mathcal{G}_{n}$ with $d$ vertical dominoes for some $d \geq 0$, construct the associated lattice path $\Phi(T)$, and factorize it as in (5.1). Since the tableau $T$ is not a fixed point, by the conditions (G1) and (G2), we observe that if the initial segment $\alpha_{0}$ of $\Phi(T)$ is empty then $d \geq 2$. We create a path $\beta$ from $\Phi(T)$ according to the following cases.

Case 1. The segment $\alpha_{0}$ is not empty. Then $\alpha_{0}$ is a Dyck path. Find the first down step going from the line $y=1$ to the $x$-axis, say $z_{a}$. Then the path $\beta$ is obtained from $\Phi(T)$ by replacing $z_{a}$ by an up step, which goes from the line $y=1$ to the line $y=2$.

Case 2. The segment $\alpha_{0}$ is empty. Then $d \geq 2$ and find the last up step going from the line $y=1$ to the line $y=2$, say $z_{b}$. Then the path $\beta$ is obtained from $\Phi(T)$ by replacing $z_{b}$ by a down step, which goes from the line $y=1$ to the $x$-axis.

Then the requested tableau $\Gamma(T)$ is defined to be the tableau associated with $\beta$, i.e., $\Gamma(T)=\Phi^{-1}(\beta)$.

Example 5.5. Given the tableau $T \in \mathcal{B}_{15}$ shown in Figure 5.2(a), the path $\Phi(T)$ is shown in Figure 5.2(b). The path $\beta$ constructed by Algorithm $\mathrm{C}$ is shown in Figure 5.1(b), and the corresponding tableau $\Lambda(T)$ is shown in Figure 5.1(a). 


\begin{tabular}{|c|c|c|c|c|c|c|c|c|}
\hline 1 & 2 & 4 & 5 & 9 & & 12 & 13 & 15 \\
\hline 3 & 6 & 7 & 8 & 10 & 11 & 14 & & \\
\hline
\end{tabular}

(a)

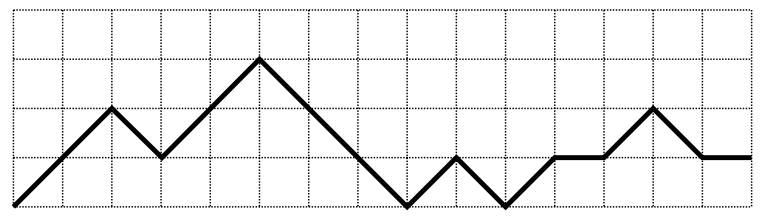

(b)

Figure 5.2: An example for the sign-reversing involution $\Gamma$ on tableaux in $\mathcal{B}_{15}$.

Proof of Theorem 1.3. (i) By Lemmas 2.2 and 5.4, we have

$$
\sum_{T \in \mathcal{R}_{2 n}} \operatorname{sign}(T)=\sum_{T \in \mathcal{B}_{n}} \operatorname{sign}(T)=\left|\mathcal{G}_{n}\right|=2\left(\begin{array}{c}
n-1 \\
\left\lfloor\frac{n-1}{2}\right\rfloor
\end{array}\right) .
$$

(ii) Let $\mathcal{B}_{n}^{(1,0)} \subset \mathcal{A}_{n}^{(1,0)}$ be the subset of $n$-domino skew SDTs with at most two rows. Notice that every tableau in $\mathcal{B}_{n}^{(1,0)}$ contains no vertical domino. Under the map $\Phi: \mathcal{A}_{n}^{(1,0)} \rightarrow$ $\mathcal{M}_{n}$ restricted to the set $\mathcal{B}_{n}^{(1,0)}$, we observe that the image of every tableau is a dispersed Dyck path of length $n$. By Lemma 5.1, we have

$$
\sum_{T \in \mathcal{R}_{2 n+1}} \operatorname{sign}(T)=\sum_{T \in \mathcal{B}_{n}^{(1,0)}} \operatorname{sign}(T)=\left(\begin{array}{c}
n \\
\lfloor n / 2\rfloor
\end{array}\right) .
$$

The proof of Theorem 1.3 is completed.

\section{Concluding remarks}

In this paper, except for the case $\mu=\left(2 e_{1}+1,2 e_{2}+1\right)$, we obtain the generating polynomials of sign-balance of the set $\mathcal{T}_{2 n}^{\left(\mu_{1}, \mu_{2}\right)}$ of $2 n$-cell skew SYTs of all shapes with at most three rows and the skew part $\mu=\left(\mu_{1}, \mu_{2}\right)$. This is accomplished by a simple sign-reversing involution on the set $\mathcal{A}_{n}^{\left(\mu_{1}, \mu_{2}\right)}$ of skew SDTs in terms of partial Motzkin paths. However, this approach is restrained by the conditions in Proposition 2.4.

For $\mu=(1,1)$, there is (essentially) a bijection between $\mathcal{A}_{n}^{(1,1)}$ and $\mathcal{M}_{n}$. If we consider the subset $\mathcal{X}_{n}^{(1,1)} \subset \mathcal{A}_{n}^{(1,1)}$ of skew SDTs with an odd number of vertical dominoes, then by the same argument as in the proof of Theorem 1.1, we have

$$
\sum_{T \in \mathcal{X}_{n}^{(1,1)}} \operatorname{sign}(T)=m_{0}+m_{1}+\cdots+m_{n-1} .
$$

The problem arises when the tableaux have an even number of vertical dominoes. For example, by Algorithm A, the two skew SDTs shown in Figure 6.1 are the images of each 
other under the involution $\Gamma: \mathcal{A}_{n}^{(1,1)} \rightarrow \mathcal{A}_{n}^{(1,1)}$. However, both of them are positive. It will be interesting to establish a sign-reversing involution on the set $\mathcal{A}_{n}^{(1,1)} \backslash \mathcal{X}_{n}^{(1,1)}$, which is helpful to solve the following question.
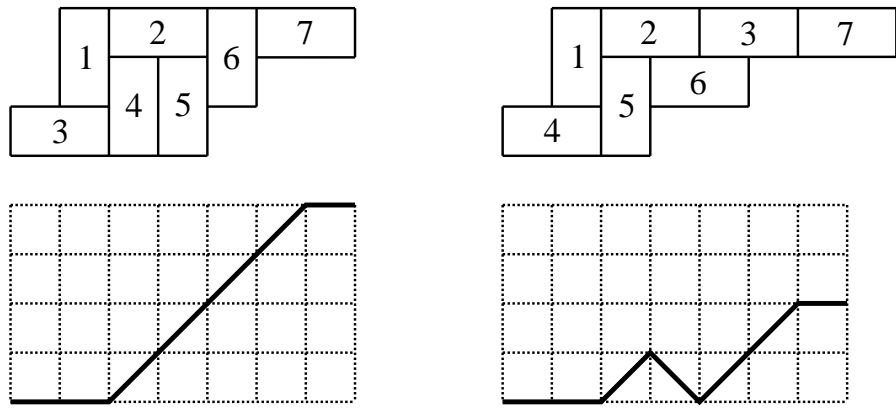

Figure 6.1: An example for the map $\Gamma$ on tableaux in $\mathcal{A}_{7}^{(1,1)}$.

Question 6.1. For $e_{1} \geq e_{2} \geq 0$, find the generating polynomial for the sign-balance of the set $\mathcal{T}_{2 n}^{\left(2 e_{1}+1,2 e_{2}+1\right)}$.

Once the sign-balance of $\mathcal{T}_{2 n}^{\left(\mu_{1}, \mu_{2}\right)}$ for the four cases of the skew part are completed, we can determine the sign-balance of $(2 n+1)$-cell skew SYTs as follows.

For any tableau $T \in \mathcal{T}_{2 n+1}^{\left(\mu_{1}, \mu_{2}\right)}$, if we remove the entry 1 from $T$ and subtract the other entries by 1 , then the resulting tableau is in $\mathcal{T}_{2 n}^{\left(\mu_{1}+1, \mu_{2}\right)}\left(\mathcal{T}_{2 n}^{\left(\mu_{1}, \mu_{2}+1\right)}\right.$ and $\mathcal{T}_{2 n}^{\left(\mu_{1}-1, \mu_{2}-1\right)}$, respectively) if the entry 1 of $T$ is in row 1 ( 2 and 3 , respectively). It follows that

$$
\begin{aligned}
\sum_{T \in \mathcal{T}_{2 n+1}^{\left(\mu_{1}, \mu_{2}\right)}} \operatorname{sign}(T)= & \sum_{T \in \mathcal{T}_{2 n}^{\left(\mu_{1}+1, \mu_{2}\right)}} \operatorname{sign}(T)+\chi\left(\mu_{1}>\mu_{2}\right) \sum_{T \in \mathcal{T}_{2 n}^{\left(\mu_{1}, \mu_{2}+1\right)}} \operatorname{sign}(T) \\
& +\chi\left(\mu_{2}>0\right) \sum_{T \in \mathcal{T}_{2 n}^{\left(\mu_{1}-1, \mu_{2}-1\right)}} \operatorname{sign}(T) .
\end{aligned}
$$

\section{Acknowledgments}

The authors thank the referees for carefully reading the manuscript and providing helpful suggestions. The first author was supported in part by Ministry of Science and Technology (MOST) grant 109-2115-M-153-004-MY2.

\section{References}

[1] R. M. Adin and Y. Roichman, Enumeration of standard Young tableaux, available in arXiv:1408.4497.

[2] T.-Y. Cheng, S.-P. Eu, T.-S. Fu and Y.-L. Lee, Skew standard domino tableaux and partial Motzkin paths, Ann. Comb. 21 (2017), no. 1, 43-71. 
[3] T. Lam, Growth diagrams, domino insertion and sign-imbalance, J. Combin. Theory Ser. A 107 (2004), no. 1, 87-115.

[4] _ On Sjöstrand's skew sign-imbalance identity, arXiv:math/0607516.

[5] A. Reifegerste, Permutation sign under the Robinson-Schensted correspondence, Ann. Comb. 8 (2004), no. 1, 103-112.

[6] B. E. Sagan and R. P. Stanley, Robinson-Schensted algorithms for skew tableaux, J. Combin. Theory Ser. A 55 (1990), no. 2, 161-193.

[7] J. Sjöstrand, On the sign-imbalance of partition shapes, J. Combin. Theory Ser. A 111 (2005), no. 2, 190-203.

[8] _ On the sign-imbalance of skew partition shapes, European J. Combin. 28 (2007), no. 6, 1582-1594.

[9] N. J. A. Sloane, The On-Line Encyclopedia of Integer Sequences, published online at http://oeis.org.

Tung-Shan Fu

Department of Applied Mathematics, National Pingtung University, Pingtung 900391, Taiwan

E-mail address: tsfu@mail.nptu.edu.tw

Chien-Tai Ting

Center for General Education, Chinese Air Force Academy, Kaohsiung 820008, Taiwan E-mail address: jazztct@gmail.com 